\title{
Subjective Wellbeing in China: A Review
}

\author{
Gareth Davey $\cdot$ Ricardo Rato
}

Published online: 26 April 2011

(c) Springer Science+Business Media B.V. 2011

\begin{abstract}
We critically review studies of subjective wellbeing conducted in China by the International Wellbeing Group, and we evaluate the International Wellbeing Index (IWI), a new instrument they developed. Subjective wellbeing was positive and similar in studies across China, and conformed to the normative range. Its resilience (PWI $=61.2-67.1$ ) mirrors survey findings conducted in Western countries, in agreement with Subjective Wellbeing Homeostasis. Reliability, validity and psychometric analyses support the utility of the IWI as a measure of subjective wellbeing. Our conclusions have implications for research and social development in China, discussed further in this review.
\end{abstract}

Keywords China $\cdot$ Happiness · International wellbeing index ·

Subjective wellbeing · Quality of life

\section{Introduction}

This article critically reviews studies of subjective wellbeing (SWB) conducted in China by the International Wellbeing Group (IWBG). It also evaluates the utility of the International Wellbeing Index (IWI), a new instrument they developed. The IWBG is a community of scholars in 50 countries working together to measure and understand SWB. The group was founded in 2001 by renowned Professor Robert Cummins, and is headquartered at the Australian Centre on Quality of Life (ACQOL) at Deakin University.

The main aim of the IWBG is to develop and utilise the International Wellbeing Index, a new measure of SWB developed by Cummins, which has been employed extensively

\footnotetext{
G. Davey $(\varangle)$

Counselling and Psychology Department, Hong Kong Shue Yan University, Hong Kong, People's Republic of China

e-mail: davey@hksyu.edu

R. Rato

School of Management, Leadership and Government, University of Saint Joseph, Macau, People's Republic of China

e-mail: ricardorato@usj.edu.mo
} 
across the globe. IWBG membership comprises three categories: Primary Researcher, responsible for gathering data in their locality; Discussant, to advance the IWI in specific areas of expertise; and Project Researcher, to use the IWI in a particular study. The IWBG's website (www.deakin.edu.au/research/acqol/iwbg) lists its membership, conferences, correspondence, documents, etc. Most communication among members is online, and there is an annual IWGB Symposium held at the International Society for Quality of Life Studies (ISQOLS) conference.

An exciting and recent development is research by the IWBG in China. This emerging literature - ranging from studies in Tibet in the north, to Guangdong and Hong Kong in the South-reveals interesting findings about SWB in China, and represents extensive evaluation of the IWI. In this article, we critically review these studies. For the purpose of our review, we use the term SWB as a generic term to denote the subjective dimension of life quality. This sidesteps the major challenge of no universal definition or single unifying theory of SWB. A wide range of terms - such as happiness, life satisfaction, positive and negative affect, subjective wellbeing, etc.-have been used in the literature. Moreover, these terms, although often assumed to be universal across cultures, can conjure different meanings, particularly in China and the Chinese language (Lau et al. 2005).

\section{International Wellbeing Index}

\subsection{Overview}

The IWI consists of two subscales: Personal Wellbeing Index (PWI) and National Wellbeing Index (NWI; Table 1). They are answered on an 11-point end-defined Likert scale, anchored from completely dissatisfied (0) to completely satisfied (10). The PWI measures SWB (personal wellbeing) via eight life-domains (community life, health, life achievement, living standard, personal relationships, safety, security, and religiosity/spirituality). Domains scores are averaged to yield a PWI score representative of the question: 'How satisfied are you with your life as a whole?'. The PWI is based on the Comprehensive Quality of Life Scale (ComQol; Cummins et al. 1994), developed as an improvement to its conceptual structure and factorability. The NWI measures satisfaction with conditions in a country, region, or city-i.e. national wellbeing - via six domains (business, economic situation, environment, government, security, and social conditions), averaged to yield a NWI score. That the NWI concerns general issues, not of direct personal concern, studies have reported national wellbeing to be lower than personal wellbeing (Tiliouine et al. 2006).

\subsection{Advantages}

The IWI was designed to be a universal measure of SWB. Each item embodies a broad aspect of life - a parsimonious approach amenable across samples, countries and cultures. It has been translated into numerous languages including Algerian, Arabic, Chinese (Cantonese, Mandarin, Tibetan), Croatian, Dutch, English, Italian, Japanese, Mexican, Norwegian, Persian, Russian, Slovakian, Spanish, etc.

Positive psychology research, being a relatively new field, is characterized by a plethora of recently-developed instruments. For example, the ACQOL website (www.acqol. deakin.edu.au) lists hundreds of scales purporting to measure SWB in one form or another; however, many contain an idiosyncratic mixture of dependent variables, failing to 
Table 1 International Wellbeing Index (English version)

\begin{tabular}{ll}
\hline Personal Wellbeing Index (PWI) & Domains \\
\hline How satisfied are you with...? & \\
1. Your standard of living? & Standard of living \\
2. Your health? & Personal health \\
3. What you are achieving in life? & Life achievement \\
4. Your personal relationships? & Personal relationships \\
5. How safe you feel? & Personal safety \\
6. Feeling part of your community? & Community connections \\
7. Your future security? & Future security \\
8. Your spirituality or religion? & Spirituality, religion \\
\hline National Wellbeing Index (NWI) & Domains \\
\hline
\end{tabular}

How satisfied are you with...?

1. The economic situation in [country]?

Economic situation

2. The state of the natural environment in [country]?

Natural environment

3. The social conditions in [country]?

Social conditions

4. Government in [country]?

Government

5. Business in [country]?

Business

6. National security in [country]?

National security

The IWI consists of two subscales: Personal Wellbeing Index (PWI) and National Wellbeing Index (NWI) An additional, optional Item 'satisfaction with life as a whole' is sometimes added to the PWI (as the first item, prior to PWI items) to test construct validity

separate clearly objective and subjective dimensions. Research in China is no exception: Chen and Davey's review of SWB research in China found that Chinese scholars used various scales, the majority being Chinese translations and modifications of Western scales (Chen and Davey 2008a). About $10 \%$ of the studies they reviewed employed scales developed in China - unknown to the international academic community-including the SWB Scale for Chinese Citizens (SWBS-CC); Chinese Adolescent Student's Life Satisfaction Scale (CASISS); and the Multiple Happiness Questionnaire (MHQ: Miao 2003; Xing and Huang 2007; Zheng et al. 2004; Chen and Davey 2008a). On the one hand, this diversity is useful because instruments can be chosen according to study aims, sample, socio-cultural context, etc. Further, concurrent utility of multiple measures offers a range of perspectives (Diener and Biswas-Diener 2000). For these reasons, we encourage our colleagues to continue their creative and innovative approaches. However, there are also drawbacks of this diverse array of instruments. Scales differ in their characteristics, such as question wording, number and type of items, rating format, instructions, etc.; and have mixed psychometric quality. This variation across studies makes it difficult to draw conclusions about SWB. With this in mind, a universal SWB measure, such as the IWI, enables comparison of findings across studies.

There are different versions of the PWI for specific subgroups: the general adult population (PWI-A); school-age children and adolescents (PWI-SC); preschool age children (PWI-PS); and adults with a cognitive impairment such as intellectual disability (PWI-ID). They have similar domain items and theoretical basis, but there are also slight modifications. The PWI-ID, for example, includes simpler item wording, and reduced-choice formats (5-, 3-, and 2-point scales) for respondents unable to cope with the standard 11-point 
scale, and a pre-testing protocol to exclude respondents unable to respond validly (McGillivray et al. 2009; Cummins et al. 2010). These parallel versions permit comparison of SWB across subpopulations.

The IWI's domain-level representation of SWB-in which scale items refer to specific life domains-is regarded by some researchers (e.g. Cummins 1996) as an improvement over single-item scales. Cummins proposed the IWI as the minimal set of domains he believes forms the first-level deconstruction of SWB (Cummins 1996; International Wellbeing Group 2006). These domains were identified during creation of the ComQol, following a review of domain names in the literature, and subsequent empirical validation (Cummins 1997; Cummins et al. 1994).

\subsection{Administration Procedure and Analyses}

The IWI has a simple, standardized administration procedure. Pilot work is usually conducted prior to a study to ensure its suitability. When testing is part of a research project, its aims, methods, possible outcomes, potential benefits and risks are fully explained, along with an overview of rights with respect to declining or agreeing to participate, and ethical considerations such as informed consent and anonymity. The researcher obtains ethics approval from the relevant authority under which they are working, such as university ethics committees, etc.

Participant's understanding of the response mode is checked. The IWI is administered verbally or in written format, with no time limit. Additional questions are oftentimes included, usually demographic profiling and the additional item 'satisfaction with life as a whole' to test construct validity. Further detail on administration procedure is available from the IWI manual (International Wellbeing Group 2006).

Prior to analysis, data are adjusted to eliminate outliers and response sets. Descriptive statistics summarize domain ratings; reliability methods include split-half reliability, Cronbach $\alpha$, test-retest, etc.; and validity is determined via factor or principal components analysis and regression against the additional item 'satisfaction with life as a whole'. The final step is conversion of all scores into a standard 0-100 scale format (into units of percentage of the scale's maximum) to compare data with other studies and general population norms.

\subsection{Theoretical Underpinning}

The IWI is embedded in 'Subjective Wellbeing Homeostasis', a theoretical framework developed by Professor Robert Cummins. This theory is summarised here briefly in order to evaluate it later in our review, although we refer readers to detailed discussions by Cummins and Nistico (2002) and Cummins et al. (2002b).

Subjective Wellbeing Homeostasis posits that SWB does not vary freely over the 0-100 range; and is maintained within a normative range (normative SWB) by a homeostatic system of internal psychological processes and external resources (Fig. 1). The normative range has a set threshold above which people maintain positive feelings about themselves, and below which SWB is negative and homeostasis has failed. Normative SWB is defended from threats to wellbeing by mechanisms such as: (1) resources external to the person-e.g. money and relationships that mediate life's challenges; and, (2) internal psychological mechanisms-e.g. processes of adaptation, selective-attention, and cognitive-restructuring — which provide habituation, restorative to peace of mind. 


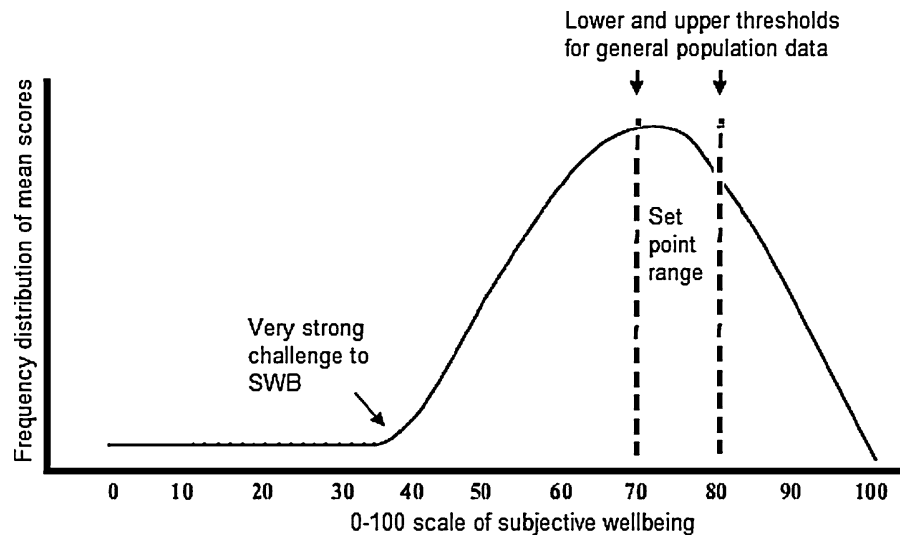

Fig. 1 Subjective Wellbeing Homeostasis, the theoretical underpinning of the IWI. It posits that selfreported SWB does not freely vary over a $0-100$ range; instead it is maintained within a narrow range by a homeostatic system of internal psychological processes and external resources. The set-point-range is 70-80 points for Western populations, and 60-80 for non-Western populations. A detailed discussion is provided by Cummins and Nistico (2002), and Cummins et al. (2002)

Empirical support for the existence of the normative range was provided by Cummins's $(1995,1998)$ analyses of SWB in several regions. His findings indicate that, on a 0-100 measurement scale, SWB among the general population is positioned within a range of approximately 70-80 points for Western populations and 60-80 for non-Western populations (a cultural response bias was identified as a plausible explanation for the difference between Western and non-Western countries). For the IWI, this means that self-reported PWI scores, when converted to a scale of 0-100, will average at about 65 (non-Western countries). Chen and Davey (2008b) replicated Cummin's methodology using datasets from various Chinese societies and found that SWB conforms to these approximations.

Under normal living circumstances, when people are not living in adverse conditions, and basic needs are being met, the theory predicts that SWB is within the normative range. Homeostasis should maintain normative SWB, although the system is under pressure from life's challenges, which might result in some variation in the range, depending on the nature and strength of threats and defense. However, in adverse living circumstances, homeostasis can be defeated, with SWB below threshold (e.g. Tiliouine et al. 2006; Fig. 1).

\section{Subjective Wellbeing in China}

This section reviews studies of the IWI conducted in China by the IWBG. Huang and Xing (2005) set the scene with the first published study in mainland China using the IWI. One of the authors, Professor Zhan-jun Xing, now based at Shandong University, is well known in China as a leading scholar and writer on SWB. In their public survey $(N=739)$ in Shangdong (an eastern coastal province on the lower reaches of the Yellow River) their findings revealed a PWI score of $65.0(\mathrm{SD}=15.8)$ and NWI score of $53.1(\mathrm{SD}=20.2)$, indicating a moderate, positive level of SWB. The IWI demonstrated good psychometric performance. Their study is important because it validated the IWI for the first time in China, which paved the way for further research. 
Building on these findings, Chen and Davey (2009) conducted a public survey in Zhuhai city, Guangdong Province $(\mathrm{n}=449)$. Zhuhai is more affluent than many other Chinese cities because it is a Special Economic Zone (SEZ) with economic, government, and legal measures conducive to business and foreign investment. Consequently, the city underwent marked economic development since the 1980s, and is now a modern, urban metropolis. Interestingly, the SWB reported by residents in Zhuhai (PWI $=64.4$; NWI $=57.4$ ), and psychometric performance of the IWI, are similar to Huang and Xing's findings. Thus, these studies show that residents in urban China seem to be satisfied with their lives; and that the IWI is appropriate.

However, social science research in China is limited by focus on urban samples, overlooking rural areas where $60 \%$ of the population reside. To remedy this issue, Davey, Chen and Lau (2009) investigated the SWB of Chinese farmers in rural Hunan, southeastcentral China. This is an interesting study because Chinese farmers are one of the country's poorest groups, with low socio-economic circumstances (Dreyer 2000; Perry \& Selden 2000; National Bureau of Statistics 2007). There is a marked divide in objective wellbeing between rural and urban areas. For example, when the study was conducted, the national average income (per person per quarter) was 4,323 RMB in urban households and 1,261 RMB in rural households; for Hunan province, it was 4,072 RMB versus 1,235. Infrastructure and access to basic services such as health and education are limited in rural areas (Wu 2007). Other rural-urban differences include emphasis in rural areas on traditional attitudes and behaviour, in contrast to more liberal, modern lifestyles in cities; and social hierarchy, as farmers are considered by some Chinese to have a lower social status (Davey 2008a; b). Whilst objective indicators such as income have often been used to characterize rural Chinese, little is known about their SWB. Davey et al. (2009) conducted a door-todoor and street survey $(\mathrm{N}=277$; ages $18-69)$ in a farming community using the PWI and qualitative interviews. The results revealed a mean PWI score of 64.2 (6.4), and domain ratings of 57.8-69.9 (SD: 15.8-24.0), similar to those listed above. Interviews with participants revealed no obvious compromise to life quality in the domains probed by the PWI. An underlying theme was that the rural folk seem content with their living standards: they felt that they had enough to satisfy their needs, despite low life quality in objective terms. Also, the study revealed that middle-aged and elderly respondents' comparison of today's situation with the past-relative standards evaluations (social and temporal comparison; Festinger 1954; Albert 1977; Michalos 1985; Diener et al. 2002)—partly explained their contentment with life, as poverty has declined markedly in recent decades.

Webb (2009) conducted the first study of SWB among Tibetan nomads ( $n=102)$, another group in China experiencing hardship. Agriculture and herding of domestic animals (cattle, goats, horses, sheep, etc.) has been the Tibetan way of life for millennia. Conditions on the remote Tibetan Plateau are challenging, including severe climate, access to only basic services, limited employment opportunities, minimal income, etc. Even so, Webb reported that the nomads are generally happy with life $(\mathrm{PWI}=70.6)$. Although the nomadic lifestyle is not so comfortable, it provides basic needs. During 5 years of his visits to the study site, the author witnessed marked improvements in infrastructure (e.g. health services, power, roads, schools, etc.). Furthermore, based on their Buddhist faith, the nomads regard their circumstances with a degree of positivity, and accept life with a peaceful mind, interpreting hardships as a progressive step to enlightenment. The author recommended a more detailed study of these tentative explanations.

Ingrid Nielsen, Russell Smyth and colleagues at Monash University in Australia have conducted excellent studies in urban China. Smyth et al. (2010) administered the PWI to residents in six Chinese cities (Chengdu, Dalian, Fushun, Fuxin, Fuzhou and Wuhan; 
$\mathrm{N}=3,390$ ), an improvement over previous studies limited to single cities and small samples. The mean PWI score for all cities was 67.1, and domain scores above 60, suggestive, again, of a moderate and positive level of SWB. Disappointingly, however, the authors reported one SWB score for all cities combined, and did not report or compare data across cities.

Nielsen et al. (2010b) studied SWB among 525 rural-urban migrant workers in Fujian Province. This is an important line of enquiry because rural-urban migration in China occurs on a large scale, involving an estimated 80-120 million migrants; it fuels the country's development and modernization, as migrants constitute the majority of the workforce in some sectors, e.g. construction. However, the migrants face many difficulties in cities. In particular, they do jobs that local people refuse, with long hours and low pay, and their housing is often sub-standard in crowded apartments, factory dormitories, and shanty towns. As their official registration is in their rural hometown, migrants are not regarded as city dwellers and, consequently, are excluded from social welfare. Moreover, migrants are sometimes scapegoats for problems such as urban crime, public health issues, etc. For these reasons, they encounter discrimination and hostility from the local population and government (Solinger 1999; Li 2008). Despite these challenges, Nielsen et al. (2010b) reported a PWI score of 62.6 (SD = 14.6), which, although slightly lower than data reported in other studies, indicates positive wellbeing. The authors suggested that the circular nature of migration - whereby migrants unable to cope in cities have the option of returning home- -buffers the adversity they face. Perhaps a more obvious explanation, not mentioned by the authors, is that conditions in the city are perceived by migrants to be better than in rural areas. Push factors for the migration are rural poverty, strenuous farming work, and unemployment; whereas pull factors include job opportunities, higher income, and an improved standard of living (Dai et al. 2006). Furthermore, although a lot more needs to done, in recent years the Chinese government has shown a more positive attitude toward migrants, including supportive policies such as better working conditions, legal rights, access to healthcare, education, etc.

Nielsen et al. (2010a) surveyed Beijing taxi drivers $(n=480)$, a group well-known in China for their long working hours (about $13 \mathrm{~h}$ per day, 7 days per week). Again, the findings (PWI score $=61.1 ; \mathrm{SD}=15.2$ ) indicate moderate SWB. The lowest domain score (Future Security, $\mathrm{M}=44.4 ; \mathrm{SD}=27.4$ ) was attributed to: job insecurity, decreased popularity of taxi services because of higher fares (increasing oil prices); and pressure from the government to adopt cleaner vehicle technologies. However, as explained later, this domain of the PWI is probably not as important in the sample as the authors reason. It is low-ranking in most of the IWBG's studies in China. This could be a reflection of the conceptual level at which the PWI operates, rather than specific to Beijing taxi drivers.

The IWI has also been studied in China's Special Administrative Regions, Hong Kong and Macau. These former European colonies, with different culture, economies and socities, are interesting contexts in which to investigate SWB. Under the "one country, two systems" principle, Hong Kong and Macau have a higher degree of autonomy than elsewhere in China (including, for example, more social freedom, separate economic and political systems). Importantly, they have a high level of objective wellbeing, including advanced economic development and living standards. There are, however, similarities to mainland China, especially in terms of culture: Hong Kong and Macau were once part of Guangdong Province, and have a shared cultural background such as language, cuisine, etc. These linkages have, of course, strengthened since transfer of sovereignty to China. Based on these differences and similarities, it is instructive to probe the SWB of people in Hong Kong and Macau, and to compare data to studies in mainland China. 
Dr. Anna Lau, formerly at the Hong Kong Polytechnic University, is a champion of research on the IWI in Hong Kong. Lau et al. (2005) compared the equivalence of the PWI in Hong Kong $(\mathrm{N}=180)$ and Australia $(\mathrm{N}=180)$. The scores of the PWI (Hong Kong, 65.9; Australia, 78.8) and domains (Hong Kong: 57.5-72.0; Australia: 74.4-83.7) differed significantly between countries, all lower in Hong Kong. The health and standard of living domains contributed to SWB more in Hong Kong than Australia. Why did the Australian respondents report higher SWB? The authors suggest a cultural response bias. However, in our view, a cultural response bias is perhaps not surprising, as it is well documented in the social science literature that Chinese respondents are more likely to choose moderate scores in psychometric scales. Therefore, future research can employ more robust crosscultural research methodology—-such as standardization, checking test construct invariance via structural equation modeling, etc.- to account for cross-cultural response bias.

Their survey also included an additional item, 'satisfaction with happiness', as previous research showed that general positive affect explains a proportion of variance in SWB, and, in psychology, although 'happiness' and 'satisfaction' are closely related concepts, the latter represents both a cognitive and affective component (Okun and Stock 1987; McDowell and Newell 1996). The item was included in the ComQol, the PWI's predecessor, but omitted in the PWI to simplify conceptual structure. Interestingly, Lau et al. (2005) found that the item explained unique variance in Australia but not Hong Kong. They suggested cultural differences in perception of 'satisfaction' and 'happiness'. For example, in traditional Chinese beliefs, as well as present-day conversation about wellbeing, the terms 'happiness' and 'satisfaction' are not always differentiated clearly. Future research is needed to explore these terms and their influence, if any, on SWB.

Further validation of the PWI in Hong Kong was conducted by Lau et al. (unpublished), involving a test-retest reliability analysis with a 2-week period. The PWI scores were 69.01 (10.18) and 69.95 (11.34) respectively, and good psychometric performance further confirmed its utility.

Lau et al. (2008) measured the public's SWB $(n=460)$ during the Severe Acute Respiratory Syndrome (SARS) pandemic. People living in districts known to have cases of SARS reported SWB (PWI = 67.1, SD = 13.5) comparable to Lau et al. (2005), suggesting no decline during the pandemic, although ratings of community-connectedness and future security domains were significantly higher among elderly respondents during the SARS outbreak. This discrepancy was explained on the basis that: 1) elderly people in Hong Kong were targeted by community outreach services and other interventions, which might have enhanced their sense of community-connectedness; and, 2) cultural factors, as the majority of older residents in Hong Kong, unlike younger residents, are from mainland China where community bonding is perhaps valued more (Bond and Hewstone 1988). Also, it is worth noting that other studies have reported social cohesion as a likely important buffer to SWB following threats experienced by a community (e.g. Liem and Liem 1978; Cummins et al. 2002a).

The Macau Special Administrative Region is a small city $\left(29.2 \mathrm{~km}^{2}\right)$ in southeast China, located on the western-side of the Pearl River Delta, adjacent to Guangdong Province. It was a Portugese colony from the 16th Century to 1999 and, therefore, has a different culture and society than Hong Kong and mainland China. The official languages are Chinese (Cantonese) and Portuguese. Rato and Davey (in press) reported their findings of a longitudinal study of SWB in Macau. The Macau Quality of Life Report was established in 2007 by the University of St. Joseph, and its methodology includes the IWI. Data were collected via quarterly public surveys (2007-2009; $\mathrm{n}=8,230)$. For all surveys combined, the PWI score was 64.4 , and domain scores were $59.4(\mathrm{SD}=17.8)$ to $69.4(\mathrm{SD}=15.8)$. 
The NWI score was $57.4(\mathrm{SD}=16.1)$, and domain scores ranged $55.2(\mathrm{SD}=18.6)$ to 65.3 $(\mathrm{SD}=15.6)$. This suggests that the general public in Macau is generally satisfied with life and conditions in the region. Lowest scores were reported in the first quarter of 2009-a period of great economic uncertainty in Macau and the world-but not significantly different to other quarters. A comparison of these data with adjacent cities (Hong Kong and Zhuhai; Lau et al. 2005; Chen \& Davey 2009) reveals remarkable similarity, despite obvious differences across cities in objective life quality.

\section{Discussion}

The studies we reviewed reveal interesting findings. SWB (PWI scores) was positive and similar in all samples, in agreement with the literature that SWB is stable and the majority of people feel happy and good about themselves (Diener and Diener 1996; Cummins 1995, 1998). Furthermore, SWB conforms to the normative range, in agreement with Chen and Davey (2008b).

Though the studies had different locations, samples, times, etc., their PWI scores are similar. Notwithstanding Webb's study in Tibet, based on a low sample size, PWI scores ranged $61.2-67.1$, a $5.9 \%$ difference. The stability of SWB is also demonstrated in the Macau Quality of Life Report, as PWI scores between 2007 and 2009 varied 3.7 points (Rato and Davey, in press). This mirrors the findings of large-scale IWI surveys conducted in Australia by Robert Cummins over a ten-year period (Cummins et al. 2007; Cummins et al. 2010). That SWB is remarkably stable, positive, and in a narrow range, supports Subjective Wellbeing Homeostasis, the IWI's theoretical underpinning.

Our review is an insight into relations between objective and subjective life quality. SWB was normative in samples with varied socio-economic variables. For example, PWI scores in Hong Kong (67.1), Macau (64.4) and Zhuhai (64.4) are similar and normative, but these cities have different culture, society and wealth. Interestingly, Davey et al. (2009) reported normative SWB among rural peasants, even though they are very poor. Respondents said they have enough to satisfy their lives-necessities such as food, housing, supportive family and community groups, etc. Other studies report that improved living standards in China have had minimal influence on SWB. For example, Gallup's nationwide surveys show no increase in SWB, even though objective life quality has improved during the same period. The poll also showed that the emerging middle class in China, despite their privileged situation, is not particularly satisfied with their income, standard of living, job, and life in general. It seems that those living the high life are not necessarily happier.

Further research needs to explain this happiness paradox in China. A recent study suggests relative deprivation-many people in China have improved their economic situation in absolute terms, but their relative income position deteriorated, due to rising income inequality (Brockmann et al. 2009). This fits findings in the West that wellbeing relates to gaps between income and material aspirations, as people become unhappy when their aspirations grow more than their incomes, despite large income increases (Frey and Stutzer 2002; Van Praag and Frijters 1999; Graham and Pettinato 2002). Also, it is well known that money and materialism can bring costs as well as benefits. It is important to emphasise however, that objective-subjective relations cannot be analysed thoroughly in China, due to preliminary status of research. Existing data can provide at best only preliminary insights. 
Subjective Wellbeing Homeostasis posits that challenges to SWB are buffered by internal and external mechanisms. Studies in China found buffers to homeostatic defeat. For example, Davey et al. (2009) describe strong family and community supporteveryone in the village knew and cared about each other-as emotional and physical security that ease life's challenges. Nielsen et al. (2010a) explained normative SWB reported by Beijing taxi drivers on personal relationships. Nielsen et al. (2010b) reasoned that migrant's challenges were cushioned by the circular nature of migration, as they can return home when life becomes too difficult.

Interestingly, none of the studies we reviewed report PWI scores below the normative range. Although China is a developing society, its situation today contrasts to widespread insecurity and poverty in the past. After 1949, economic planning improved quality of life, except during the bad years of 1959, 1960, and 1961. Hundreds of millions of people have been lifted out of poverty; mortality rates decreased markedly; and urban areas have transformed via infrastructure development. Since the 1980s, increasing living standards, income and consumerism have enabled people to afford products and services conducive to a satisfying life. However, a significant number of Chinese are still poor and lack access to necessities: A notable disparity, for example, is rich provinces in the east coast versus poor central and western China. Research is needed in poor provinces such as Gansu, Guizhou, Tibet, Qinghai, etc. which lag far behind in objective indicators.

All studies in China by the IWBG used the seven-item and not the eight-item PWI. In 2006 the IWBG agreed to probe the addition of the spirituality/religiosity domain. Research in Colombia among Christians (Wills 2009) and Algeria among Muslims (Tiliouine 2009) supports its inclusion. However, the new domain has not been evaluated in China, presumably because it was not part of the IWI until recently. We have been reluctant to use it because most people in mainland China do not officially adhere to a religion, and it is sometimes controversial. In our trial studies in Macau, $40 \%$ of respondents responded to the eight item. Nonetheless, further research could investigate it further in China. We also suggest research on the PWI-SC, PWI-PS, PWI-ID, and NWI, as most studies hitherto employed only the PWI-A.

Research in China by the IWBG can be seen as an extensive psychometric evaluation of the IWI. In all studies, detailed reliability and validity analyses revealed good psychometric performance, summarized in Table 2. Principal components and factor analyses revealed two factors/components with strong loadings, as PWI and NWI items loaded onto their respective components. Variance explained by the PWI, and each domain's contribution to unique variance, showed the PWI items to constitute the minimum domain set representing the first-level deconstruction of 'Life as a whole' (in most studies, standard of living made the largest contribution, followed by life achievement). These are welcome findings because it is desirable, of course, to use reliable and valid instruments (Diener 2006). There is also consistency in ranking of domain scores, as the relationships, health, and safety domains were highest ranked; whereas community connection, security, and life achievement lowest. For the NWI, regional security was highest, whereas environment lowest. It is difficult to explain this finding without further research. It might be an indication of the stable nature of SWB, a reflection of the conceptual level at which the PWI and NWI operate, or for other reasons. It should be noted that the validation of the IWI for Chinese populations is a work in progress, and more studies are needed. Other methods are needed to evaluate the IWI; for example, no studies have yet tested convergent and divergent validity.

Another limitation is that some studies report only PWI and NWI scores, accompanied by psychometric analyses. We question their worth, and recommend studies to probe SWB in 


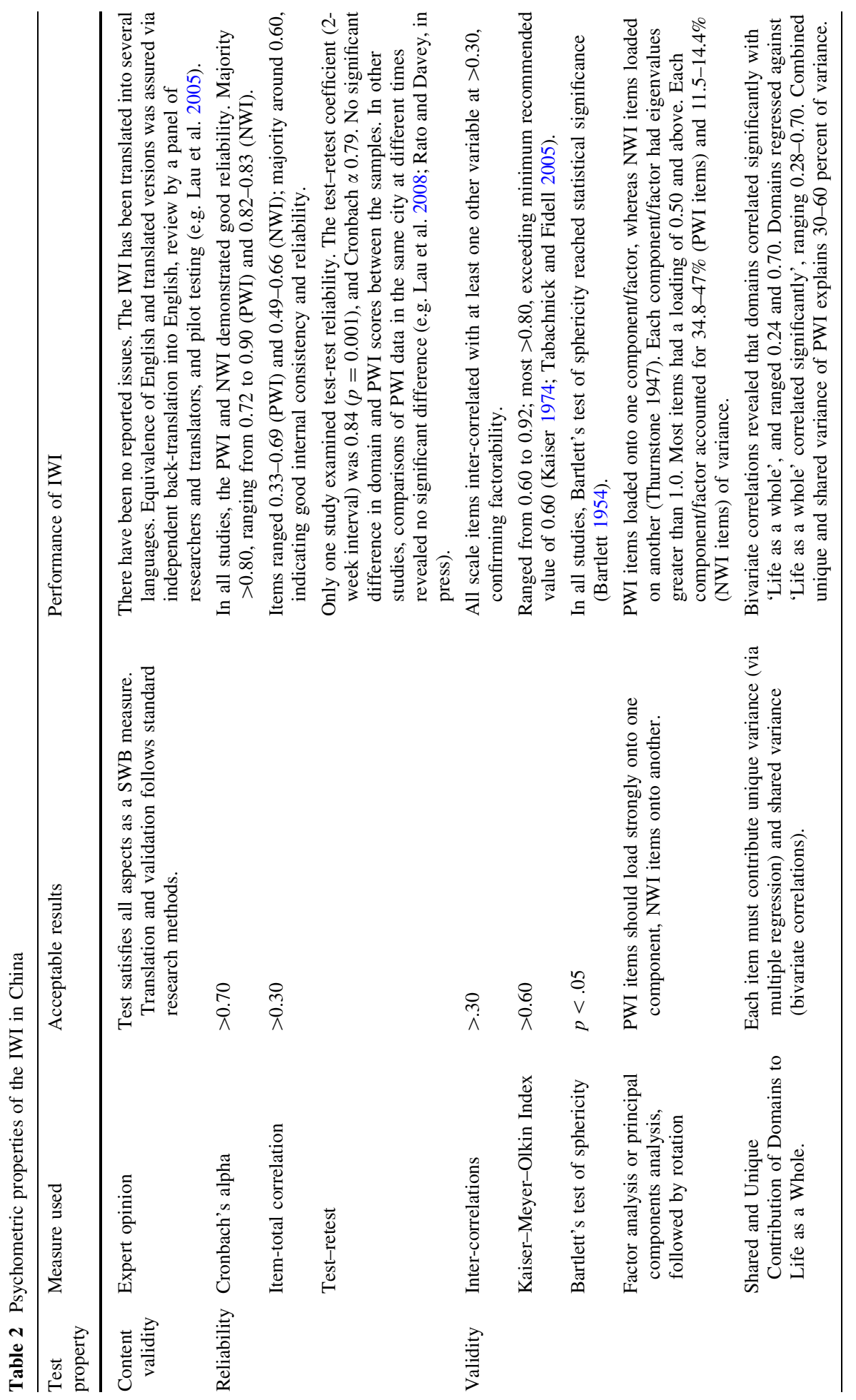


greater depth. Studies worldwide show that many factors interact with SWB. Research on SWB in China has investigated mediators such as acculturation, age, personality, social grouping, social support, etc. Other factors impinging on SWB include human rights issues such as discrimination, freedom of speech, one-child policy, politics, religion, worker's rights, etc.

\section{Implications for Social Development}

The findings in this review have implications for China's development. The first is to conduct more studies, particularly public surveys. As shown here, there is a paucity of research in most provinces and cities. This limits our understanding of SWB and, therefore, applied benefits of social indicators research ranging from strategic planning and policymaking to public debate about the best societal conditions to live in. We suggest the setting up of projects similar to The Macau Quality of Life Report, which, to our knowledge, is the only long-term and large-scale survey of SWB in China. The development of national and regional SWB surveys is also at an early stage in the West, as only a handful of regional programmes have been developed. Examples include the Australian Unity Wellbeing project in Australia; Eurobarometer in the European Union; Latinobarometer in Chile; and Afrobarometer surveys in Africa. International surveys include the World Values Survey, International Social Survey, etc.

Chinese policy makers and politicians need to understand SWB. Dialogue on development in China fails to appreciate subjective indicators. Economists and policy makers emphasize tangible measures such as economic prosperity, overlooking happiness. In contrast to the preliminary status of SWB research in China, there is voluminous economic data. The National Bureau of Statistics of China, for example, regularly publishes sophisticated datasets. Since the 1980s, thousands of books and periodicals have been published on the Chinese economy. Economic development is a priority because of the assumption that wealth elevates personal wellbeing. Money enables people to meet their needs and to afford resources conducive to a satisfying life. Also, richer people can afford resources to overcome challenges (Kahneman and Krueger 2003; Schwartz 2004; Varian 1992; Diener and Seligman 2004). But development should not be based only on wealth, measured by economic indicators, but also on people's SWB. Discrepancies between economic indices and subjective measures shed doubt on economic progress as a barometer of wellbeing. The failure of policy makers to appreciate SWB as a social indicator hinders the development of policies to enhance population happiness.

\section{References}

Albert, S. (1977). Temporal comparison theory. Psychological Review, 84, 485-503.

Bartlett, M. S. (1954). A note on the multiplying factors for various x2 approximations. Journal of the Royal Statistical Society, 16, 296-298.

Bond, M. H., \& Hewstone, M. (1988). Social identity theory and the perception of ingroup relations in Hong Kong. International Journal of Intercultural Relation, 12, 153-170.

Brockmann, H., Delhey, J., Welzel, C., \& Yuan, H. (2009). The China puzzle: Falling happiness in a rising economy. Journal of Happiness Studies, 10(4), 387-405.

Chen, Z., \& Davey, G. (2008a). Happiness and subjective wellbeing in China. Journal of Happiness Studies, 9(4), 589-600.

Chen, Z., \& Davey, G. (2008b). Normative life satisfaction in Chinese societies. Social Indicators Research, $89(3), 557-564$. 
Chen, Z., \& Davey, G. (2009). Subjective quality of life in Zhuhai City, South China: A public survey using the international wellbeing index. Social Indicators Research, 91(2), 243-258.

Cummins, R. (1995). On the trail of the gold standard for subjective wellbeing. Social Indicators Research, 35, 179-200.

Cummins, R. (1996). The domains of life satisfaction: An attempt to order chaos. Social Indicators Research, 38, 303-332.

Cummins, R. (1998). A second approximation to an international standard for life satisfaction. Social Indicators Research, 43, 307-334.

Cummins, R., Lau, A., Davey, G. \& McGillivray, J. (2010). Measuring subjective wellbeing: The personal wellbeing index intellectual disability. In R. Kober (Ed.) Enhancing the quality of life of people with intellectual disability: from theory to practice. Berlin: Springer.

Cummins, R., Woerner, J., Tomyn, A., Gibson, A., \& Knapp, T. (2007). Australian unity wellbeing index survey 17. Australia: Australian Centre on Quality of Life.

Cummins, R., Woerner, J., Weinberg, M., Perera, C., Gibson-Prosser, A., Collard, J., et al. (2010b). Australian unity wellbeing index survey 24. Australia: Australian Centre on Quality of Life.

Cummins, R. A., McCabe, M. P., Romeo, Y., \& Gullone, E. (1994). The comprehensive quality of life scale (ComQol): Instrument development and psychometric evaluation on tertiary staff and students. Educational and Psychological Measurement, 54, 372-382.

Cummins, R. A. (1997) Assessing quality of life. In R. Brown (Ed.), Assessing quality of life for people with disabilities (pp. 116-150). Cheltenham, UK: Stanley Thornes.

Cummins, R.A., Gullone, E., \& Lau, A.L.D. (2002). A model of subjective wellbeing homeostasis: The role of personality. In E. Gullone and R. A Cummins (Eds.), The universality of subjective wellbeing indicators: Social Indicators Research Series (pp. 7-46). Dordrecht: Kluwer.

Cummins, R. A., \& Nistico, H. (2002). Maintaining life satisfaction: The role of positive cognitive bias. Journal of Happiness Studies, 3, 37-69.

Cummins, R. A., Eckersley, R., Pallant, J., \& Davern, M. (2002a). The international wellbeing group and the Australia unity wellbeing index. Social Indicators Network News, 69, 8.

Dai, T., Davey, G., \& Higgins, L. (2006). Rural-urban migration in China. Geography Review, 19(5), $2-6$.

Davey, G. (2008a). Avoidance and fear by Chinese adults by age, ethnic group, sex, and residency. Psychological Reports, 103, 23-34.

Davey, G. (2008b). Children's television, radio, Internet, and computer usage in a city and a village of China. Visual Anthropology, 21(2), 160-165.

Davey, G., Chen, Z., \& Lau, A. (2009). Peace in a thatched hut-that is happiness: Subjective wellbeing among peasants in rural China. Journal of Happiness Studies, 10(2), 239-252.

Diener, E. (2006). Guidelines for national indicators of subjective well-being and ill-being. Journal of Happiness Studies, 7, 397-404.

Diener, E., \& Biswas-Diener, R. (2000). New directions in subjective wellbeing research: The cutting edge. Indian Journal of Clinical Psychology, 27, 21-33.

Diener, E., \& Diener, C. (1996). Most people are happy. Psychological Science, 7, 181-185.

Diener, E., Lucas, R. E., Oishi, S., \& Suh, E. M. (2002). Looking up and looking down: Weighting good and bad information in life satisfaction judgments. Personality and Social Psychology Bulletin, 28, 437-445.

Diener, E., \& Seligman, M. (2004). Beyond money: toward an economy of wellbeing. Psychological science in the public interest, 5(1), 1-31.

Dreyer, J. (2000). China's political system: Modernization and tradition. London: Macmillan.

Festinger, L. (1954). A theory of social comparison processes. Human Relations, 7, 117-140.

Frey, B., \& Stutzer, A. (2002). Happiness and economics: How the economy and institutions affect human wellbeing. Princeton, NJ: Princeton University Press.

Graham, C., \& Pettinato, S. (2002). Frustrated achievers: Winners, losers, and subjective wellbeing in new market economies. Journal of Development Studies, 38(4), 100-140.

Huang, 1., \& Xing, Z. (2005). An initial research on the wellbeing index applied to citizens in China. Chinese Journal of Behavioral Medical Science, 114(5), 464-465.

International Wellbeing Group. (2006). Personal wellbeing index. Australia: Australian Centre on Quality of Life.

Kahneman, D., \& Krueger, A. (2003). Developments in the measurement of subjective wellbeing. Journal of Economic Perspectives, 20(1), 3-24.

Kaiser, H. F. (1974). An index of factor simplicity. Psychometrika, 39, 31-36.

Lau, A., Chi, I., Cummins, R., Lee, T., Chou, K., \& Chung, L. (2008). The SARS (Severe Acute Respiratory Syndrome) pandemic in Hong Kong: Effects on the subjective wellbeing of elderly and younger people. Aging \& Mental Health, 12(6), 746-760. 
Lau, A., Cummins, R., \& McPherson, W. (2005). An investigation into the cross-cultural equivalence of the personal wellbeing index. Social Indicators Research, 72, 403-430.

Lau, A., Davey, G. \& Cummins, R. (unpublished). Test-retest reliability of the personal welling index (PWI) in Hong Kong.

$\mathrm{Li}$, B. (2008). Why do migrant workers not participate in urban social insurance schemes? The case of the construction and service sectors in Tianjin. In I. Nielsen \& R. Smyth (Eds.), Migration and social protection in China. Singapore: World Scientific.

Liem, R., \& Liem, J. (1978). Social class and mental illness reconsidered: The role of economic stress and social support. Journal of Health and Social Behavior, 19, 139-156.

McDowell, L., \& Newell, C. (1996). Measuring health: Guide to rating scales and questionnaires. New York: Oxford University Press.

McGillivray, J., Lau, A., Cummins, R., \& Davey, G. (2009). The utility of the 'Personal Wellbeing IndexIntellectual Disability Scale (PWI-ID)' in an Australian sample. Journal of Applied Research in Intellectual Disabilitiesl, 22, 276-286.

Miao, Y. (2003). Happiness in psychology filed: Research into the theory and measurement of well-being. Unpublished Ph.D. thesis, Nanjing Normal University (in Chinese).

Michalos, A. (1985). Multiple discrepancies theory (MDT). Social Indicators Research, 16, 347-413.

National Bureau of Statistics. (2007). China statistical yearbook. Beijing, China: National Bureau of Statistics.

Nielsen, I., Paritski, O., \& Smyth, R. (2010a). Subjective wellbeing of Beijing taxi drivers. Journal of Happiness Studies, 11(6), 721-733.

Nielsen, I., Smyth, R., \& Zhai, Q. (2010b). Subjective wellbeing of China's off-farm migrants. Journal of Happiness Studies, 11, 315-333.

Okun, M. A., \& Stock, W. A. (1987). Correlates and components of subjective well-being among the elderly. The Journal of Applied Gerontology, 6(1), 95-112.

Perry, E., \& Selden, M. (Eds.). (2000). Chinese society: Change, conflict and resistance. London: Routledge.

Rato, R., \& Davey, G. (in press). Quality of life in Macau, China. Social indicators research.

Schwartz, B. (2004). The paradox of choice: Why more is less. New York: Ecco.

Smyth, R., Nielsen, I., \& Zhai, Q. (2010). Personal wellbeing in urban China. Social Indicators Research, 95, 231-251.

Solinger, D. (1999). Contesting citizenship in urban China: Peasant migrants, the state and the logic of the market. Berkeley, CA: University of California Press.

Tabachnick, B. G., \& Fidell, L. (2005). Using multivariate statistics (5th ed.). London: Pearson.

Tiliouine, H. (2009). Measuring satisfaction with religiosity and its contribution to the personal well-being index in a muslim sample. Applied Research in Quality of Life, 4(1), 91-108.

Tiliouine, H., Cummins, R. A., \& Davern, M. (2006). Measuring wellbeing in developing countries: The case of Algeria. Social Indicators Research, 75, 1-30.

Van Praag, B., \& Frijters, P. (1999). The measurement of welfare and wellbeing: The Leyden approach. In D. Kahneman, E. Diener, \& N. Schwaraz (Eds.), Wellbeing: The foundations of hedonic psychology (pp. 413-433). New York: Russell Sage Foundation.

Varian, H. (1992). Microeconomic analysis. New York: Norton.

Webb, D. (2009). Subjective wellbeing on the Tibetan Plateau: An exploratory investigation. Journal of Happiness Studies, 10, 753-768.

Wills, E. (2009). Spirituality and subjective well-being: Evidences for a new domain in the personal wellbeing index. Journal of Happiness Studies, 10, 49-69.

Wu, T. (2007) Urban-rural divide in china continues to Widen. Gallup. Retrieved from: http://www.gallup. com/poll/27028/urbanrural-divide-china-continues-widen.aspx.

Xing, Z., \& Huang, L. (2007). A study on subjective quality of life among current Chinese primary social groups. Social Sciences in Nanjing, 1, 83-97. (in Chinese).

Zheng, X., Sang, D., \& Wang, L. (2004). Acculturation and subjective wellbeing of Chinese students in Australia. Journal of Happiness Studies, 5, 57-72. 